\title{
ESTILOS DE PENSAMIENTO EN ALUMNOS DE QUINTO AÑO DE SECUNDARIA DE DIFERENTES NIVELES SOCIOECONÓMICOS DE LA CIUDAD DE LIMA
}

\author{
THINKING STYLE IN STUDENTS OF FIFTH YEAR OF SECONDARY FROM DIFFERENT \\ SOCIOECONOMIC LEVELS OF THE CITY OF LIMA \\ TOMÁs CAYCHO R. ${ }^{1}$ \\ Fundación Felipe Antonio Custer, Lima, Perú \\ (RECIBIDO EL 11/01/2010, ACEPTADO EL 08/06/2010)
}

\begin{abstract}
RESUMEN
Se realizó un estudio descriptivo comparativo con el objetivo de explorar diferencias en los estilos de pensamiento en una muestra de adolescentes estudiantes de quinto año de educación secundaria de Lima Metropolitana provenientes de diferentes niveles socioeconómicos. Los instrumentos utilizados para el estudio fueron el Cuestionario de estilo de pensamiento de Sternberg-Wagner forma corta, y la escala socioeconómica. Los resultados muestran que los estilos de pensamiento mejor valorados son el legislativo, externo y liberal, mientras que los estilos menos valorados son el estilo oligárquico, el interno y el conservador. Se identificaron diferencias entre los alumnos de diferentes niveles socioeconómicos en cuanto a los estilos de pensamiento legislativo, ejecutivo, monárquico, anárquico, jerárquico, oligárquico, global, local, interno, externo, liberal y conservador. Así mismo, en la comparación por género se identificaron diferencias significativas entre varones y mujeres en los estilos legislativo, ejecutivo, judicial, monárquico, jerárquico, local, externo, liberal y conservador.
\end{abstract}

Palabras clave: Estilo de pensamiento; nivel socioeconómico; adolescentes.

\begin{abstract}
We performed a comparative descriptive study aimed to explore differences in thinking styles in a sample of adolescent students in fifth year of secondary education in Metropolitan Lima from different socioeconomic levels. The instruments used for the study were the questionnaire Sternberg's Thinking Style - Wagner Short Form, and the Socioeconomic Scale. The results show that thinking styles are rated as legislative, external and liberal, while less valued styles are style oligarchic, internal and the conservatives. Differences were found between students of different socioeconomic levels in terms of thinking styles legislative, executive, monarchist, anarchist, hierarchical, oligarchic, global, local, internal, external, liberal and conservative. Likewise, in the gender comparison, significant differences were found between men and women in styles legislative, executive, judicial, monarchist, hierarchy, local, external, liberal and conservative.
\end{abstract}

Keywords: thinking style; socioeconomic status; adolescents.

1 Psicólogo de la Universidad Nacional Mayor de San Marcos. E-mail: tonyvoxdei1783@hotmail.com. 


\section{INTRODUCCIÓN}

En la actualidad encontramos diversas investigaciones que informan acerca de la relación entre las aptitudes de los alumnos y el rendimiento académico, pero no todas coinciden en relación a la amplitud de sus resultados. En general, los resultados de la investigación correlacional indican la existencia de una relación moderada (sobre 0.50) entre aptitud y rendimiento (González-Pienda J., Núñez J., Gonzales-Pumariega S., Álvarez L., Roces C., Gonzáles P., Bernardo A., Valle A., Cabanach R., Rodríguez S. y Sales P., 2004). Aún teniendo como referencia las investigaciones referidas al nivel de globalidad/ especificidad entre ambas variables, la aptitud de los alumnos únicamente es capaz de explicar entre un $25 \%$ y un $33 \%$ de la varianza del rendimiento académico (Neisser et al., 1996; en González-Pienda J., Núñez J., Gonzales-Pumariega S., Álvarez L., Roces C., Gonzáles P., Bernardo A., Valle A., Cabanach R., Rodríguez S. y Sales P., 2004). Dos o más alumnos pueden presentar un patrón semejante en cuanto a sus destrezas o habilidades intelectuales, pero pueden presentar estilos de pensamiento diferentes que afectarían las diferencias académicas entre ambos. Es así que los estilos de pensamiento no se encuentran ni en el dominio de las habilidades, ni en el de la personalidad, sino en el espacio de interacción entre ambos (Sternberg, 1997).

Sternberg (1999) propone la teoría del "autogobierno mental" como modelo de estilos de pensamiento. Este modelo se basa en un paralelismo entre la organización del individuo y la organización de la sociedad. En primer lugar, de la misma manera que la sociedad necesita gobernarse así misma también las personas necesitamos autogobernarnos, como los gobiernos también las personas necesitan establecer prioridades y distribuir recursos, las personas necesitan responder a los cambios del mundo como los gobierno y, de la misma manera que es difícil cambiar los gobiernos, también lo es cambiarnos a las personas (Sternberg, 1999).

Para Sternberg, los estilos de pensamiento son las vías preferidas de utilizar y aplicar la propia inteligencia a un problema o labor que se ha de completar. Estas formas características de pensar juegan un papel importante en la adaptación de las personas a su medio ambiente, pues representan la manera en que ellas emplean sus recursos cognitivos para manejar sus ambientes y adaptarse (Escurra, 2001). Comprender la forma cómo las personas piensan, brindará información para un mejor conocimiento personal y para saber si es que se están aprovechando al máximo sus capacidades.

Sternberg propuso 13 estilos de pensamiento, los cuales derivan de los tipos de gobierno que existen en el mundo. De igual manera que los gobiernos, las personas, para resolver problemas y adaptarse a su medio, necesitan ejercen ciertas funciones mediante los estilos legislativo, ejecutivo y judicial. La puesta en marcha de estas funciones se puede llevar a cabo de diversas formas, caracterizadas por los estilos monárquico, jerárquico, oligárquico y anárquico; en diferentes niveles, como los estilos global y local; en diversos alcances, como los estilos interno y externo, y con determinadas inclinaciones, ya sea liberal o conservador (Aguilar y Morales, 2007).

Las funciones de los estilos de pensamiento hacen referencia al tipo de actividad que desempeñan las personas en el estudio, trabajo o en cualquier actividad diaria para buscar adaptarse a su sociocultura. Así tenemos: a) el estilo legislativo, caracterizado por la 
preferencia a crear fórmulas y planear soluciones a los problemas más que seguir las establecidas, poniendo en tela de juicio las normas y suposiciones antes que aceptarlas (Delgado, 2004); b) el estilo ejecutivo, caracterizado por seguir reglas o actividades en las que les especifican lo que deben y cómo lo deben hacer (Sternberg, 1997), así como manejar problemas previamente estructurados y planteados, y c) el estilo judicial, tendiente a realizar comparaciones, contrastar, evaluar, corregir y juzgar ideas, procedimientos, y problemas ya existentes.

Las formas de los estilos están referidas a la manera diferente y particular de afrontar el mundo y los problemas que se pueden presentar en él, ya sea tomando en cuenta una única perspectiva, varias perspectivas, o de una manera aleatoria y desorganizada. Así tenemos: a) el estilo monárquico, caracterizado por abordar los diferentes problemas desde una sola perspectiva, es decir que toman en cuenta un solo objetivo o necesidad, poniendo énfasis en aquellos que más le interesan; b) el estilo jerárquico, caracterizado por establecer una jerarquía de metas, reconociendo la necesidad de examinar los problemas desde varios puntos de vista para establecer correctamente las prioridades y distribuir sus recursos con prudencia; c) el estilo oligárquico, caracterizado por enfrentar, al igual que el estilo jerárquico, los problemas desde múltiples puntos de vista, pero tomando en consideración un conjunto de objetivos y metas consideradas de igual importancia que en ocasiones pueden resultar contradictorias entre sí, y d) el estilo anárquico, tendiente a abordar de manera aleatoria los problemas, utilizando varios procedimientos para su solución, pero carentes de normas o reglas que puedan sustentar sus prioridades.

Los niveles de los estilos se conciben como las diferentes formas de abordar los problemas para darles una pronta solución, ya sea de manera general o particular. Así tenemos: a) el estilo global, caracterizado por abordar y dar solución a los problemas tomando en cuenta cuestiones amplias y abstractas, tendiendo a ignorar o rechazar los detalles presentes en la situación problemática, y b) el estilo local, tendiente a enfrentar un problema partiendo de la consideración de los detalles y de las cuestiones concretas.

El alcance de los estilos se refiere al tipo de interacción que efectúan las personas ya sea consigo mismas (mundo interno) o con los demás (mundo externo). El estilo interno se caracteriza por el gusto por situaciones en las que pueden llevar a cabo sus propias ideas sin recurrir a nadie, así como discutir cuestiones utilizando solo sus puntos de vista (Sternberg, 1999). Mientras que el estilo externo está caracterizado por la orientación a la gente y la expansión (Sternberg, 1999), la conciencia de lo que le sucede a otras personas, así como la discusión y el trabajo en grupo (Delgado, 2004).

Las inclinaciones de los estilos hacen mención a las diversas tendencias de buscar o evitar el cambio a la hora de enfrentar los diferentes problemas o cuestiones que se presentan en su sociocultura. El estilo liberal está caracterizado por ir más allá de los procedimientos y reglas existentes, maximizando el cambio y buscando situaciones que sean algo ambiguas, prefiriendo algún grado de incertidumbre en la vida y en el trabajo (Delgado, 2004). El estilo conservador trata de evitar el cambio, buscando antiguos procedimientos para dar solución a los problemas, ciñéndose a situaciones familiares en el trabajo y en la vida, evitando situaciones ambiguas siempre que sea posible (Delgado, 2004). 
Un gran número de investigaciones como las de Rojas, Salas y Jiménez (2006); Dai, D. y Feldhusen, J. (1999); Kim, J. y Michael, W. B. (1995); Rosário, P., Nuñez, J., Gonzáles-Pienda, J., Almeida, L., Soares, S. y Rubio, M. (2005); Zhan, L. y Sachs, J. (1997) han demostrado la utilidad de los estilos de pensamiento como variable predictora en el rendimiento escolar.

Por otro lado, existe gran cantidad de evidencia sobre la relación entre los procesos psicológicos y los niveles socioeconómicos a que pertenecen los individuos, pero las relaciones específicas entre estas variables aún no han recibido la suficiente atención por parte de los psicólogos. La investigación sobre factores relacionados con el desarrollo cognitivo, a nivel internacional y en el Perú, ha mostrado repetidamente la importancia del nivel socioeconómico de los estudiantes, tanto individualmente como agrupados en aulas (Cuetos y Rodríguez, 2003; Ruiz, Rosales y Neira, 2006).

Es necesario precisar que en el presente trabajo utilizamos el término nivel socioeconómico para designar el lugar donde se sitúa un individuo en la sociedad, en función del ingreso económico de la familia, la educación de los padres, bienes y servicios, materiales de construcción de la vivienda, lugar de residencia en la ciudad y las condiciones de habitabilidad.

Parte de los contenidos representacionales es originada por el mismo individuo a través de su propio esfuerzo, mientras que otra parte le es transmitida por otros individuos mediante procesos comunicativos (tales como la educación y la ideología en general). En otro sentido, unos de esos contenidos son relativamente exclusivos del individuo ("conocimiento personal") mientras que otros son compartidos o comunes a grupos de individuos ("conocimiento colectivo", referido a una cultura) (Padrón, 2008). Es así que las personas se diferencian y se asemejan entre sí por el modo particular en que opera su función cognitiva, debido, entre otras cosas, a que la realidad circundante (contexto socioeconómico) no es la misma para todos. A pesar de la expansión educativa, las desigualdades culturales y económicas se siguen combinando para formar desigualdades en los niveles de educación (Benavides, 2004).

Para los maestros y demás profesionales involucrados en la enseñanza, el conocimiento de los estilos de pensamiento y su comportamiento en diferentes niveles socioeconómicos implica hacer que la enseñanza sea más eficaz en el logro de sus objetivos en busca de la equidad social. Según Sternberg (1999), el principio básico para que los estudiantes se beneficien al máximo de la enseñanza y la evaluación reside en que al menos parte de estas deben armonizar con sus estilos de pensamiento (Delgado, 2004).

El conocimiento de la importancia de los estilos de pensamiento y la influencia de las condiciones socioeconómicas de su familia de origen nos plantea la siguiente interrogante:

¿Existen diferencias significativas en los estilos de pensamiento de alumnos de quinto grado de educación secundaria provenientes de diferentes niveles socioeconómicos? 


\section{MÉTODO}

\section{Diseño de la investigación}

El diseño de la investigación corresponde al descriptivo comparativo, en donde se realizará la medición de los estilos de pensamiento, comparando los resultados de la medición con el nivel socioeconómico y el sexo al que pertenecen los estudiantes (Alarcón, 2008; Sánchez y Reyes, 2006).

\section{Población y muestra}

La población corresponde a la totalidad de alumnos matriculados en el quinto de secundaria de diversos centros educativos de Lima Metropolitana, según estadísticas reportadas por el Ministerio de Educación del Perú. La muestra fue determinada mediante un procedimiento no aleatorio por conveniencia y está constituida por 600 escolares, varones y mujeres, de quinto año de educación secundaria de centros educativos públicos y privados de Lima Metropolitana.

Tabla N. ${ }^{\circ}$ 1. Composición general de la muestra de alumnos evaluados.

\begin{tabular}{|c|c|c|c|}
\hline & Variable & $\mathbf{F}$ & $\%$ \\
\hline \multirow{11}{*}{ Edad } & 15 años & 147 & 24.5 \\
\hline & 16 años & 224 & 37.4 \\
\hline & 17 años & 116 & 19.4 \\
\hline & 18 años & 47 & 7.8 \\
\hline & 19 años & 28 & 4.7 \\
\hline & 20 años & 8 & 1.3 \\
\hline & 21 años & 8 & 1.3 \\
\hline & 22 años & 9 & 1.5 \\
\hline & 23 años & 6 & 1 \\
\hline & 24 años & 5 & 0.8 \\
\hline & 25 años & 2 & 0.3 \\
\hline \multirow{2}{*}{ Sexo } & Varón & 306 & 51.0 \\
\hline & Mujer & 294 & 49.0 \\
\hline \multirow{2}{*}{ Tipo de colegio } & Estatal & 396 & 65.97 \\
\hline & Particular & 204 & 34.03 \\
\hline \multirow{3}{*}{ Estrato socioeconómico } & Bajo & 224 & 37.3 \\
\hline & Medio & 235 & 39.2 \\
\hline & Alto & 141 & 23.5 \\
\hline
\end{tabular}

$\mathrm{N}=600$ 


\section{Instrumentos}

Cuestionario de estilos de pensamiento de Sternberg-Wagner, forma corta (Sternberg, R., 1999). El cuestionario está constituido por 65 afirmaciones acerca de diferentes aspectos asociados a las preferencias individuales por la ejecución de diversas tareas y procesos mentales. Los enunciados se califican en un sistema tipo Likert con 7 puntos de calificación. El cuestionario puede ser aplicado de manera individual o colectiva y está diseñado para ser administrado a grupos de adolescentes y adultos por el tipo de afirmaciones que contiene. Las trece escalas que conforman el Cuestionario de estilos de pensamiento presentan coeficientes alfa de Cronbach que van desde 0.64 a 0.85 , y un coeficiente de confiabilidad generalizado alfa de Cronbach de 0.86 lo que nos indica que el mencionado cuestionario es confiable. El análisis factorial se realizó a través del método de componentes principales con rotación varimax. Los resultados permiten apreciar que el instrumento se encuentra conformado por cinco factores, que juntos explican el $67.8 \%$ de la varianza indicando que presenta validez de constructo.

Escala socioeconómica (Caycho, T., 2009), ficha de recolección de datos socioeconómicos elaborada en base a rangos de amplitud homogéneos, permitiendo así que la clasificación de un hogar en un nivel depende únicamente del puntaje alcanzado y no de un sesgo en las amplitudes. Es así que el puntaje máximo alcanzado puede ser de 24 puntos (nivel socioeconómico alto) y el mínimo 5 puntos (nivel socioeconómico bajo). Es así que para que un hogar sea clasificado como del nivel socioeconómico alto deberá alcanzar una puntuación de 21 a 24 puntos, los de nivel socioeconómico medio deberán alcanzar una puntuación de 17 a 20 puntos, mientras que para ser catalogado como perteneciente al nivel socioeconómico bajo deberá obtener una puntuación de 5 a 8 puntos.

\section{Variables comparadas}

a) Estilos de pensamiento: legislativo, ejecutivo, judicial, jerárquico, anárquico, monárquico, oligárquico, global, local, interno, externo, liberal y conservador.

b) Estrato socioeconómico: alto, medio y bajo.

c) Sexo: varón y mujer.

\section{Variables controladas}

Nivel educativo: alumnos de quinto año de educación secundaria de Lima Metropolitana.

\section{RESULTADOS}

Realizando el análisis jerárquico de los estilos de pensamiento en la totalidad de la muestra (Tabla N. ${ }^{\circ} 2$ ) podemos apreciar que el estilo mejor evaluado corresponde al estilo legislativo $(\mathrm{M}=5.45$, D. E. $=0.65)$, seguido del estilo externo $(\mathrm{M}=5.33$, D. $\mathrm{E} .=0.95)$ y el estilo liberal $(M=5.30$, D. $E .=0.70)$, en segundo y tercer lugar respectivamente. Por otro lado, los estilos de pensamiento menos valorados son el estilo conservador $(\mathrm{M}=4.15$, D. $\mathrm{E} .=1.08)$, interno $(\mathrm{M}=4.25$, D. E. $=0.74)$ y oligárquico $(\mathrm{M}=4.32$, D. $\mathrm{E} .=0.76)$. 
Tabla N. ${ }^{\circ}$ 2. Análisis jerárquico de los estilos de pensamiento de los estudiantes de $5 .^{\circ}$ de secundaria de Lima Metropolitana.

\begin{tabular}{clccc}
\hline & \multicolumn{1}{c}{ Escalas } & Tipo & Media & D. E.+ \\
\hline 1 & Legislativo & Función & 5.45 & 0.65 \\
2 & Externo & Alcance & 5.33 & 0.95 \\
3 & Liberal & Inclinación & 5.30 & 0.70 \\
4 & Jerárquico & Forma & 5.17 & 0.81 \\
5 & Judicial & Función & 5.14 & 0.71 \\
6 & Global & Nivel & 5.02 & 0.62 \\
7 & Ejecutivo & Función & 4.71 & 0.82 \\
8 & Local & Nivel & 4.70 & 0.72 \\
9 & Monárquico & Forma & 4.65 & 0.73 \\
10 & Anárquico & Forma & 4.35 & 0.84 \\
11 & Oligárquico & Forma & 4.32 & 0.76 \\
12 & Interno & Alcance & 4.25 & 0.74 \\
13 & Conservador & Inclinación & 4.15 & 1.08 \\
\hline \multicolumn{4}{c}{$\mathrm{n}=600$} \\
\hline
\end{tabular}

Para la contrastación de hipótesis se procedió, en primer lugar, a realizar el análisis de aproximación de las puntuaciones a la curva normal, efectuada mediante la aplicación de la prueba de Kolmogorov-Smirnov (Tabla N. ${ }^{\circ} 3$ ). El resultado nos indica que no existen diferencias significativas en los estilos de pensamiento evaluados, por lo que podemos concluir que los puntajes tienen una adecuada distribución a la curva normal. Lo anteriormente dicho permite realizar los análisis inferenciales de contrastación de hipótesis empleando estadísticos paramétricos.

Tabla N. ${ }^{0}$ 3. Prueba de bondad de ajuste a la curva normal de Kolmogorov-Smirnov.

\begin{tabular}{clccc}
\hline & Escalas & Media & D. S. & K-S Z \\
\hline 1 & Legislativo & 5.45 & 0.65 & 0.85 \\
2 & Ejecutivo & 4.71 & 0.95 & 0.89 \\
3 & Judicial & 5.14 & 0.70 & 1.32 \\
4 & Monárquico & 4.65 & 0.81 & 0.91 \\
5 & Jerárquico & 5.17 & 0.71 & 0.53 \\
6 & Oligárquico & 4.32 & 0.62 & 1.07 \\
7 & Anárquico & 4.35 & 0.82 & 1.09 \\
8 & Global & 5.02 & 0.72 & 1.41 \\
9 & Local & 4.70 & 0.73 & 0.95 \\
10 & Interno & 4.25 & 0.84 & 1.01 \\
11 & Externo & 5.33 & 0.76 & 1.34 \\
12 & Liberal & 5.30 & 0.74 & 1.26 \\
13 & Conservador & 4.15 & 1.08 & 1.07 \\
\hline & & Significativo $\mathrm{p}<0.05$ & \\
\hline
\end{tabular}


Al comparar los estilos de pensamiento según el nivel socioeconómico podemos apreciar la existencia de diferencias significativas en los estilos legislativo $(\mathrm{F}=1.58, \mathrm{Sig} .=0.03)$ y ejecutivo $(F=19.61$, Sig. $=0.04)$, monárquico $(F=7.24$, Sig. = 0.03), jerárquico $(\mathrm{F}=2.91$, Sig. $=0.02)$, oligárquico $(\mathrm{F}=21.11$, Sig. = 0.01), anárquico $(\mathrm{F}=7.12$, Sig. $=$ 0.03), global $(F=7.11$, Sig. $=0.03)$, local $(F=2.78$, Sig. $=0.02)$, interno $(F=12.05$, Sig. $=0.02)$, externo $(F=9.25$, Sig. $=0.01)$, liberal $(F=3.02$, Sig. $=0.03)$ y conservador $(\mathrm{F}=20.65$, Sig. $=0.01)$.

Tabla N. ${ }^{0}$ 4. Análisis comparativo de los estilos de pensamiento legislativo, ejecutivo y judicial de los alumnos de quinto de secundaria de Lima Metropolitana según nivel socioeconómico mediante el análisis de varianza de un factor.

\begin{tabular}{|c|c|c|c|c|c|c|c|c|c|}
\hline & \multirow{3}{*}{ Escala } & \multicolumn{6}{|c|}{ Nivel socioeconómico } & \multirow{3}{*}{$\mathbf{F}$} & \multirow{3}{*}{ Sig. } \\
\hline & & \multicolumn{2}{|c|}{ Alto } & \multicolumn{2}{|c|}{ Medio } & \multicolumn{2}{|c|}{ Bajo } & & \\
\hline & & $\mathbf{M}$ & D. E. & $\mathbf{M}$ & D. E. & $\mathbf{M}$ & D. E. & & \\
\hline 1 & Legislativo & 5.41 & 0.68 & 5.33 & 0.68 & 5.11 & 0.78 & 1.58 & $0.031 *$ \\
\hline 2 & Ejecutivo & 4.87 & 0.79 & 5.08 & 0.77 & 5.52 & 0.75 & 19.16 & $0.046^{*}$ \\
\hline 3 & Judicial & 5.15 & 0.79 & 5.12 & 0.64 & 5.15 & 0.68 & 8.19 & 0.652 \\
\hline 4 & Monárquico & 4.48 & 0.87 & 4.58 & 0.76 & 4.83 & 0.69 & 7.24 & $0.038^{*}$ \\
\hline 5 & Jerárquico & 5.11 & 0.69 & 5.18 & 0.72 & 5.51 & 0.84 & 2.91 & $0.021 *$ \\
\hline 6 & Oligárquico & 4.37 & 0.69 & 4.75 & 0.71 & 4.38 & 0.71 & 21.11 & $0.011 *$ \\
\hline 7 & Anárquico & 4.63 & 0.71 & 4.41 & 0.73 & 4.24 & 0.77 & 7.12 & $0.034 *$ \\
\hline 8 & Global & 5.31 & 0.65 & 5.27 & 0.83 & 5.02 & 0.81 & 7.11 & $0.034 *$ \\
\hline 9 & Local & 4.70 & 0.72 & 4.77 & 0.68 & 4.87 & 0.73 & 2.78 & $0.025^{*}$ \\
\hline 10 & Interno & 4.26 & 0.67 & 4.13 & 1.14 & 4.08 & 1.17 & 12.05 & $0.025^{*}$ \\
\hline 11 & Externo & 5.27 & 0.72 & 5.76 & 0.78 & 5.28 & 0.75 & 9.25 & $0.018^{*}$ \\
\hline 12 & Liberal & 5.30 & 0.85 & 5.54 & 0.81 & 4.94 & 0.61 & 3.02 & $0.035^{*}$ \\
\hline 13 & Conservador & 4.09 & 1.09 & 4.27 & 0.62 & 5.34 & 0.72 & 20.65 & $0.010^{*}$ \\
\hline
\end{tabular}

Cuando se comparan los estilos de pensamiento de los estudiantes considerando el sexo (Tabla N. ${ }^{\circ}$ ), las mujeres presentan medias más altas que los varones en los estilos legislativo $(\mathrm{T}=2.45, \mathrm{Sig} .=.024)$ y liberal $(\mathrm{T}=2.73, \mathrm{Sig} .=.028)$, mientras que los varones presentan medias más altas que las mujeres en los estilos ejecutivo $(\mathrm{T}=1.37$, Sig. $=.028)$, judicial $(\mathrm{T}=5.81, \mathrm{Sig} .=.021)$, monárquico $(\mathrm{T}=3.43, \mathrm{Sig} .=.008)$, jerárquico $(\mathrm{T}=2.54$, Sig. $=.012)$, local $(\mathrm{T}=2.57$, Sig. $=.024)$, externo $(\mathrm{T}=2.94$, Sig. $=.035)$ y conservador $(\mathrm{T}=3.71$, Sig. $=.000)$. 
Tabla N. ${ }^{\circ}$ 5. Análisis comparativo de los estilos de pensamiento legislativo, ejecutivo y judicial de los alumnos de quinto de secundaria de Lima Metropolitana según sexo.

\begin{tabular}{lcccccc}
\hline \multirow{2}{*}{ Escalas } & \multicolumn{2}{c}{ Varón } & \multicolumn{2}{c}{ Mujer } & \multirow{2}{*}{ T } & Sig. \\
\cline { 2 - 5 } & $\mathbf{M}$ & D. E. & M & D. E. & & \\
\hline Legislativo & 5.21 & 0.65 & 5.38 & 0.64 & 2.45 & $.024^{*}$ \\
Ejecutivo & 4.77 & 0.75 & 4.63 & 0.87 & 1.37 & $.028^{*}$ \\
Judicial & 5.12 & 0.64 & 4.94 & 0.71 & 5.81 & $.021^{*}$ \\
Monárquico & 4.76 & 0.65 & 4.52 & 0.73 & 3.43 & $.008^{*}$ \\
Jerárquico & 5.28 & 0.71 & 5.06 & 0.61 & 2.54 & $.012^{*}$ \\
Oligárquico & 4.27 & 0.72 & 4.18 & 0.76 & 2.55 & .51 \\
Anárquico & 5.08 & 0.56 & 5.01 & 0.67 & 2.07 & .55 \\
Global & 4.35 & 0.64 & 4.30 & 0.79 & 2.11 & .57 \\
Local & 4.78 & 0.68 & 4.61 & 0.74 & 2.57 & $.024^{*}$ \\
Interno & 4.32 & 0.81 & 4.35 & 0.87 & 1.71 & .62 \\
Externo & 5.28 & 0.84 & 5.17 & 1.03 & 2.94 & $.035^{*}$ \\
Liberal & 5.30 & 0.79 & 5.44 & 0.81 & 2.73 & $.028^{*}$ \\
Conservador & 4.33 & 1.10 & 3.07 & 1.01 & 3.71 & $.00^{*}$ \\
\hline & & $*$ Significativo p $<.05$ & & \\
\hline
\end{tabular}

\section{DISCUSIÓN}

El análisis jerárquico de los estilos de pensamiento indica que los estudiantes de quinto de secundaria de Lima Metropolitana presentan una tendencia a la creación y formulación de nuevas maneras de enfrentarse a los problemas y de sobrepasar los lineamientos preestablecidos, así como la preferencia por realizar trabajos grupales en los que se encuentre involucrado el trato interpersonal. Estas características estarían dando cuenta que la muestra estudiada tiene un fuerte potencial creativo; lamentablemente el sistema educativo actual no suele estimular a este tipo de alumnos, más bien tiende a relegarlos por considerarlos alumnos que en ciertas ocasiones pueden ser problemáticos debido a que suelen ser sumamente críticos con la enseñanza que se les imparte (Delgado, 2004). Estos últimos, muchas veces son olvidados porque se considera que, por ser creativos, no necesitan apoyo, ni un programa especial (Monroy y Ruiz, 2001). Es importante notar la importancia que los alumnos le dan al estilo externo, debido a que cuando se enfrenten al mundo laboral la gran parte de sus actividades se llevarán a cabo de manera grupal y si no se ha tenido una adecuada experiencia en trabajo de grupo el rendimiento en las actividades laborales serán bajas debido a que no se establecen relaciones significativas en busca de los objetivos a conseguir en el trabajo. Estos resultados están en concordancia con lo encontrado por Kim y Michael (1995) y Escurra (2000), quienes en estudiantes 
universitarios de primer año encontraron que estos tienen preferencia por estilos de tipo creativo e innovador.

Las comparaciones por niveles socioeconómicos a los que pertenecen los estudiantes de la muestra indican que existen marcadas diferencias en los estilos de pensamiento. Se observa que los estudiantes de niveles socioeconómicos más altos dirigen sus capacidades de manera creativa, buscando siempre nuevas maneras de abordar los problemas, mientras que los de nivel socioeconómico bajo (NSB) tienen un estilo de pensar más práctico y orientado al presente, hallazgo que concuerda con lo afirmado por Ardila (1979).

Por otro lado, los estudiantes de nivel socioeconómico alto (NSA) y medio (NSM) presentan una menor capacidad de organización y jerarquización de los objetivos, resultándoles difícil planificar sus medios de acción, en comparación a los estudiantes de NSB quienes prefieren establecer una jerarquía de prioridades y llevarlos a cabo uno por uno. El tener un estilo jerárquico, según Sternberg (1999), es una ventaja en los estudios y en el campo laboral, pues muchas de estas organizaciones favorecen este estilo de pensamiento. En el ámbito educativo, por ejemplo en lo relacionado a los cursos y a los exámenes, los estudiantes tienen que establecer prioridades en cuanto al tiempo y al esfuerzo realizado. Es pertinente señalar que en ocasiones las personas jerárquicas pueden centrarse tanto en los elementos de una jerarquía que pueden caer en la indecisión, resultando que cuando se tiene que llevar a cabo un proyecto grande puede resultar más ventajoso otro estilo, tal como por ejemplo, el monárquico (Delgado, 2004).

Por otro lado, los alumnos de NSA tienden en su mayoría a preocuparse más por cuestiones generales y abstractas, mientras que los estudiantes de NSB tienen predilección a preocuparse más por los detalles y aspectos concretos. Sternberg (1999) señala que la clave para solucionar con éxito los problemas que se presentan en diversas situaciones es la capacidad de la persona de pasar de un nivel a otro, es así que si se elige trabajar en un nivel determinado (por ejemplo, global), en el ámbito educativo o clínico, es sumamente útil formar equipos con personas que tiene predilección por el nivel contrario (local) (Delgado, 2004).

Los alumnos de NSA, en comparación de los de NSM y NSB, tienen mayor predilección por desarrollar habilidades de planificación, autocontrol y autoevaluación de trabajos, así como monitoreos de sus propios objetivos, lo que contribuirá a su desarrollo personal (Witkin, en Díaz-Guerrero, 1994). Estos alumnos, podrían presentar mayor tendencia a la introversión que sus compañeros de aula y tienden a pasar algunos periodos de tiempo solos por diferentes razonas: a) tienen dificultad en encontrar pares similares a ellos con los cuales compartir sus intereses y temperamento; b) son por lo general aislados por sus compañeros dado que los perciben como nerds, y c) están por lo general tan ensimismados en sus propios proyectos que tienen poco tiempo para socializar (Blumen, 2008).

Así mismo, los alumnos de NSM alcanzan valores más elevados en el estilo externo, evidenciando tener buenas habilidades sociales, aprendiendo mejor con motivaciones extrínsecas, pero requiriendo de ayudas externas y de constante retroalimentación por parte de otras personas (Witkin, en Díaz-Guerrero, 1994). Los alumnos provenientes del NSB presentan puntajes mayores que los de NSA y NSM, indicando que los alumnos que 
viven situaciones desfavorecidas tienden a seguir en varias ocasiones procedimientos ya conocidos, no confiando siempre en nuevos cambios que se puedan producir.

Los hallazgos en relación al nivel socioeconómico son el reflejo de las diferentes condiciones de vida de cada grupo de estudio (INEI, 1999, en Tarazona, 2005).

El análisis por sexo refleja la existencia de diferencias significativas en los estilos legislativo, ejecutivo, judicial, monárquico, jerárquico, local, externo, liberal y conservador. Estos hallazgos concuerdan con los estudios de Klatic (1999), quien observó diferencias significativas en los estilos monárquico y local; así como los estudios de Escurra (2001), que apreció diferencias significativas en los estilos judicial, externo, liberal y conservador. Observamos que los varones tienen predilección por la organización de prioridades y de realizar una actividad a la vez, así también son personas que tienden a centrarse en lo detalles y peculiaridades de los acontecimientos, siendo también personas con sensibilidad social y capacidad de establecer fácilmente relaciones sociales, prefiriendo el trabajo en grupo. Estos hallazgos coinciden con lo reportado por Miranda (1999) y Sternberg (1997, 1999) para quienes las personas con estilo legislativo o judicial tienden a ser más locales. Por otro lado, las mujeres se caracterizan por ser abiertas a nuevos procedimientos de acción para dar solución a los problemas y adaptarse así satisfactoriamente a su medio. Se observa de esta manera que la mujer tiene un gran número de oportunidades para el desarrollo de su vida emotiva y de su papel específico de feminidad (Díaz Guerrero, 1974).

Las diferencias psicológicas encontradas entre mujeres y varones corrobora la idea que el género influye diferencialmente en los rasgos psicológicos durante la adolescencia en relación con los procesos de socialización diferenciados para cada uno de los sexos.

Es pertinente aclarar que las personas no tienen un único y definitivo estilo de pensamiento, sino que todos podemos presentar en un momento u otro cada uno de los diferentes estilos.

El hallazgo de las diferencias de los estilos de pensamiento según el nivel socioeconómico constituye una nueva evidencia de los efectos de la sociocultura y de la pobreza en el desarrollo de las cogniciones, tal como lo han propuesto diversos autores (Estefanía y Tarazona, 2003; Acevedo, 1996; Alarcón, 1986; Ardila, 1979). De forma similar, la relación entre las condiciones de vida y la socialización diferenciada según género influyen en el desarrollo psicológico (Mansilla, s/f en Tarazona, 2005).

Sin lugar a dudas, podemos decir que estos resultados pueden y deben generar una serie de preguntas y cuestionamientos, lo que permitirá continuar adentrándonos en el tema. La consideración de aceptar algunas hipótesis y rechazar otras, así como la consistencia de algunos hallazgos permiten sentar bases para elaborar líneas de acción pertinentes a las diferentes realidades.

\section{CONCLUSIONES}

1. Los estilos de pensamiento mejor valorados son el estilo legislativo, externo y liberal.

2. Las mujeres presentan valores más altos que los varones en los estilos de pensamiento legislativo y liberal. 
3. Los varones presentan valores más altos que las mujeres en los estilos ejecutivo, judicial, monárquico, jerárquico, local, externo y conservador.

4. Existen diferencias significativas, según el nivel socioeconómico, en los estilos de pensamiento legislativo, ejecutivo, monárquico, jerárquico, oligárquico, anárquico, global, local, interno, externo, liberal y conservador.

\section{NOTA DE RECONOCIMIENTO}

El responsable brinda su agradecimiento al Consejo Superior de Investigaciones de la U.N.M.S.M. por su financiamiento, a las autoridades y docentes de los centros educativos en donde se ha llevado a cabo el estudio por su colaboración en la aplicación de los instrumentos y, de manera especial, a los alumnos, cuyo aporte ha permitido obtener la información necesaria para la presente investigación. Así mismo, al Dr. Carlos Arenas Iparraguirre, Dr. Jaime Aliaga Tovar, Mg. Lidia Sotelo López y Lic. David Tarazona por los aportes y sugerencias para el desarrollo de la presente investigación.

\section{REFERENCIAS BIBLIOGRÁFICAS}

1. Acevedo, I. (1996). Factores de vulnerabilidad asociados a la condición de pobreza. Un enfoque ecosistémico cognitivo. En Aprendizaje y Comportamiento, 11 (1-2), 53-72.

2. Aguilar, M. y Morales, M. (2007). Estilos de pensamiento, tipos de liderazgo y estilos educativos en docentes universitarios. En Revista de Psicología, 9, 81-91.

3. Delgado, A. (2004). Relación entre los estilos de aprendizaje y los estilos de pensamiento en estudiantes de maestría considerando las especialidades profesionales y el tipo de universidad (tesis doctoral no publicada), Lima, Perú: Facultad de Psicología de la Universidad Nacional Mayor de San Marcos.

4. Escurra, L. (2001). Estilos de Pensamiento en estudiantes de la U.N.M.S.M. En Revista de investigaciones en psicología de la U.N.M.S.M., 4 (1), 9-34.

5. González-Pienda, J.; Núñez, J.; Gonzales-Pumariega, S.; Álvarez, L.; Roces, C.; Gonzáles, P.; Bernardo, A.; Valle, A.; Cabanach, R.; Rodríguez, S. y Sales, P. (2004). Estilos de pensamiento: análisis de su validez estructural a través de las respuestas de adolescentes al Thinking Styles Inventory. En Psicothema, 16 (1), 139-148.

6. Padrón, J. (2008). Estilos de pensamiento y exclusión social. En EntreCiencias, 1 (1), 117-148.

7. Rojas, G., Salas, R. y Jiménez, C. (2006). Estilos de aprendizaje y estilos de pensamiento entre estudiantes universitarios. En Estudios pedagógicos XXXII, 1, 49-75.

8. Sternberg, R. (1997). la creatividad en una cultura conformista, un desafío a las masas. Barcelona, España: Paidós.

9. Sternberg, R. (1999). Estilos de pensamiento. Barcelona, España: Ediciones Paidós. 